\title{
Design of RF MEMS based switch matrix for space applications
}

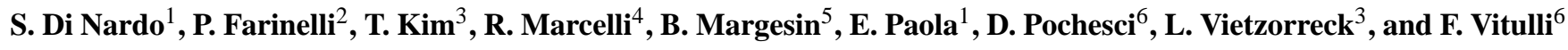 \\ ${ }^{1}$ ThalesAlenia Space, L'Aquila, Italy \\ ${ }^{2}$ University of Perugia, Perugia, Italy \\ ${ }^{3}$ Technische Universität München, Munich, Germany \\ ${ }^{4} \mathrm{CNR}$ - Institute for Microelectronics and Microsystems, Roma, Italy \\ ${ }^{5}$ FONDAZIONE BRUNO KESSLER, Trento, Italy \\ ${ }^{6}$ ThalesAlenia Space, Roma, Italy \\ Correspondence to: T. Kim (kim@tum.de)
}

\begin{abstract}
RF MEMS based switch matrices have several advantages compared to the mechanical or solid-state switch based ones for space applications. They are compact, light and less lossy with a high linearity up to high frequency. In this work, a $12 \times 12$ switch matrix with RF MEMS and LTCC technologies is presented based on the planar Beneš network. The simulated performance of the $12 \times 12$ switch matrix is below $-12 \mathrm{~dB}$ IL (Insertion Loss) up to $\mathrm{C}$ band and $-15 \mathrm{~dB}$ RL (Return Loss) up to Ku band. Moreover, it has a good isolation better than $-50 \mathrm{~dB}$. A $4 \times 4$ switch matrix with the same design process and technologies is fabricated and measured to verify the $12 \times 12$ switch matrix design process. The measured performance agrees very well to the simulations.
\end{abstract}

\section{Introduction}

In a communication satellite, the received signals are simultaneously to be delivered to the assigned transmission outputs with high flexibility, which is normally achieved by high-order switch matrices. Microwave or millimeter-wave switch matrices provide a full and high connectivity to the satellite broadcasting system. The switch matrices which are used up to now are based on the mechanical switches. The mechanical switches are reliable, less lossy and can drive a high power with high linearity. However, they are slow, bulky, heavy and require motors for switching, which are normally unsuitable for the cost-effective high speed system. Switch matrices using solid state devices, such as PIN diodes, MESFETs (MEtal Semiconductor Field Effect Transistors) or HEMTs (High Electron Mobility Transistor), have been also discussed due to their high speed and compact size with cheaper prices. However, they consume a DC power and may generate intermodulation spurious modes. Moreover, their applicable frequency bands are limited. Recently, Radio Frequency Micro Electro Mechanical System (RF-MEMS)s are referred to as a good candidate to get over the reported drawbacks of the mechanical or solid state based switches. They are small and virtually consume no power. RF MEMS can be operated up to high frequency with high linearity. Moreover, on maturing microfabrication technologies, the cost for RF MEMS fabrication is going down (De Angelis et al., 2012).

In the present paper, the design of the RF MEMS switch matrix is discussed. The aimed switch matrix has 12 inputs and 12 outputs. Starting from the network topology and the control algorithm, the design process for the $12 \times 12$ switch matrix is described including physical assembly with the control boards and the housing. Based on the results from full wave and circuit simulators, the RF performance is presented as well. A $4 \times 4$ switch matrix with same design process and technologies is fabricated and the measured performance is compared to simulations.

\section{Discussion of network topology}

Network topologies have been investigated mainly in the field of computer architecture. The representative network topologies are Crossbar, Omega, Beneš or Clos network, etc. The proper choice of the network topology for the RF MEMS networks is important because the number of required switching elements and stages makes a strong effect to the construction feasibility together with the signal transition performance and the network functionallity. Considering the required switch numbers and control algorithm implementations, Beneš and Clos networks are good candidates to 
construct a high order switch matrix using RF MEMS technologies.

A Bene ̌s network is a powerful rearrangeable network with simple control algorithms. Rearrangeable networks mean that any permutation can be statically assigned by rearranging network paths if necessary. The Beneš network is one of the oldest and best-known rearrangeable networks in use for around 40 years. It finds widespread use in shared memory-multiprocessor systems, telecommunication networks, TDMA (Time Division Multiple Access) systems for satellite communications and newer applications such as switching fabrics in internet routers. To realize a RF MEMS switch matrix, the Beneš network can be implemented in a planar way. Figure 1a shows the network block diagram for the $12 \times 12$ planar Beneš network using DPDT (Double-Pole Double-Throw) switches. A DPDT switch has two inputs and two outputs. The $12 \times 12$ Beneš network is constructed based on the $16 \times 16$ Beneš network (Chang and Melhem, 1997).

A Clos network is also a subclass of the rearrangeable networks. Such a network consists of two symmetric outside stages of rectangular switches with an inner stage of square switches. Those three-stage-arranged switch modules are interconnected with every module in the adjacent stage by a unique link (Yeh and Feng, 1992). More practical implementation to the RF MEMS switch matrix is 3D Clos-Beneš network, which is to construct small sub-matrices using SPnT (Single-Pole n-Throw) or DPDT RF MEMS switches and assemble them to the complicated Clos network as described in Fig. 1b. In the figure, it is depicted that 8 SP4T switches are used to construct a $4 \times 4$ switch sub-matrix. For an easy connection, the inner stage is $90^{\circ}$ rotated and assembled to the outer stages in a three-dimensional way.

Physically, planar Beneš and 3D Clos Beneš networks can be realized as Fig. 2. The figure also shows the estimated sizes and weights. The planar Beneš networks consist of several planar circuit boards. They are simple and easy to assemble. They are also robust with compact size. The drawback of the planar Beneš network is that the isolation can be poor because all circuits in the network are installed in one metal housing. However, the circuit coupling can be suppressed by inserting several metal vias and frames with covers. Compared to the planar Beneš network, 3D Clos Beneš networks have better isolation. They are easier to be extended to the higher order networks. The design of a single sub-matrix is also simple. However, their assembly is mechanically complex. As described in the figure, the middle stage is rotated by $90^{\circ}$ and connected to the other stages by several coaxial cables, which requires high costs and a large footprint together with complex DC control input port connects. The 3D Clos Beneš network is expected to be bigger and heavier than the planar Beneš network's. In this work, a planar Beneš network is decided to be fabricated due to its simplicity, smaller footprint and lower weight.

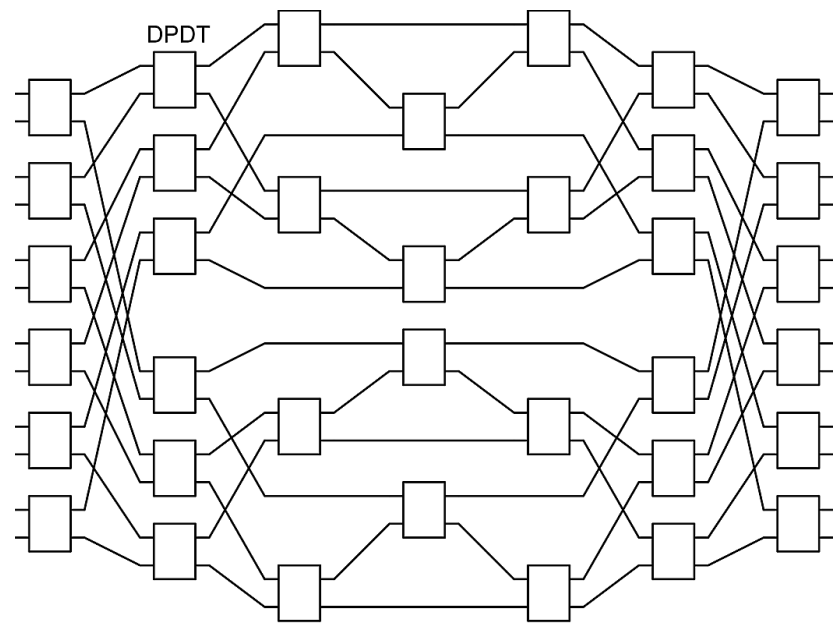

(a) Planar Benes network
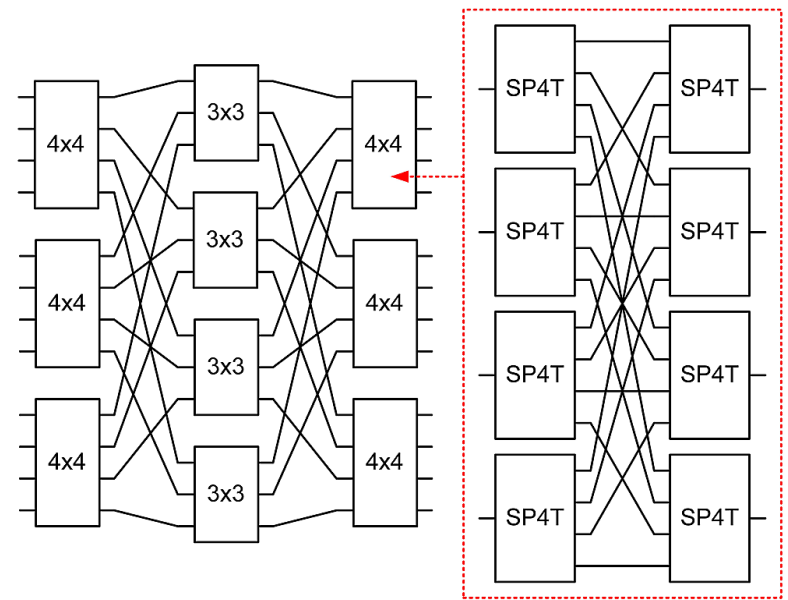

(b) 3D Clos Benes network

Fig. 1. $12 \times 12$ Planar Beneš and $12 \times 12$ 3-D Clos Beneš network.

\section{$312 \times 12$ Switching matrix}

\subsection{Switching matrix design}

In the present work, the network physically consists of 6 RF boards and 6 DC boards which are actually 3 kinds of RF boards and two types of DC boards. RF boards consist of 4 LTCC (Low Temperature Co-fired Ceramic) and 2 alumina boards. The LTCC is an attractive solution to integrate electric components for high frequency applications. Compared to the printed resin board, it has good high frequency characteristics, thermal stability and is also suitable for passive components integrations (Imanaka, 2005). The RF signal which comes to the alumina board input is transmitted to the circuit on the LTCC board. After being routed to the assigned output port of the LTCC board stages (Stage 1, Stage 2 in Fig. 3), the RF signal is transmitted to the final network output through circuits on alumina boards. The DC signals to control RF MEMS switches are applied to the network by 6 DC boards. 


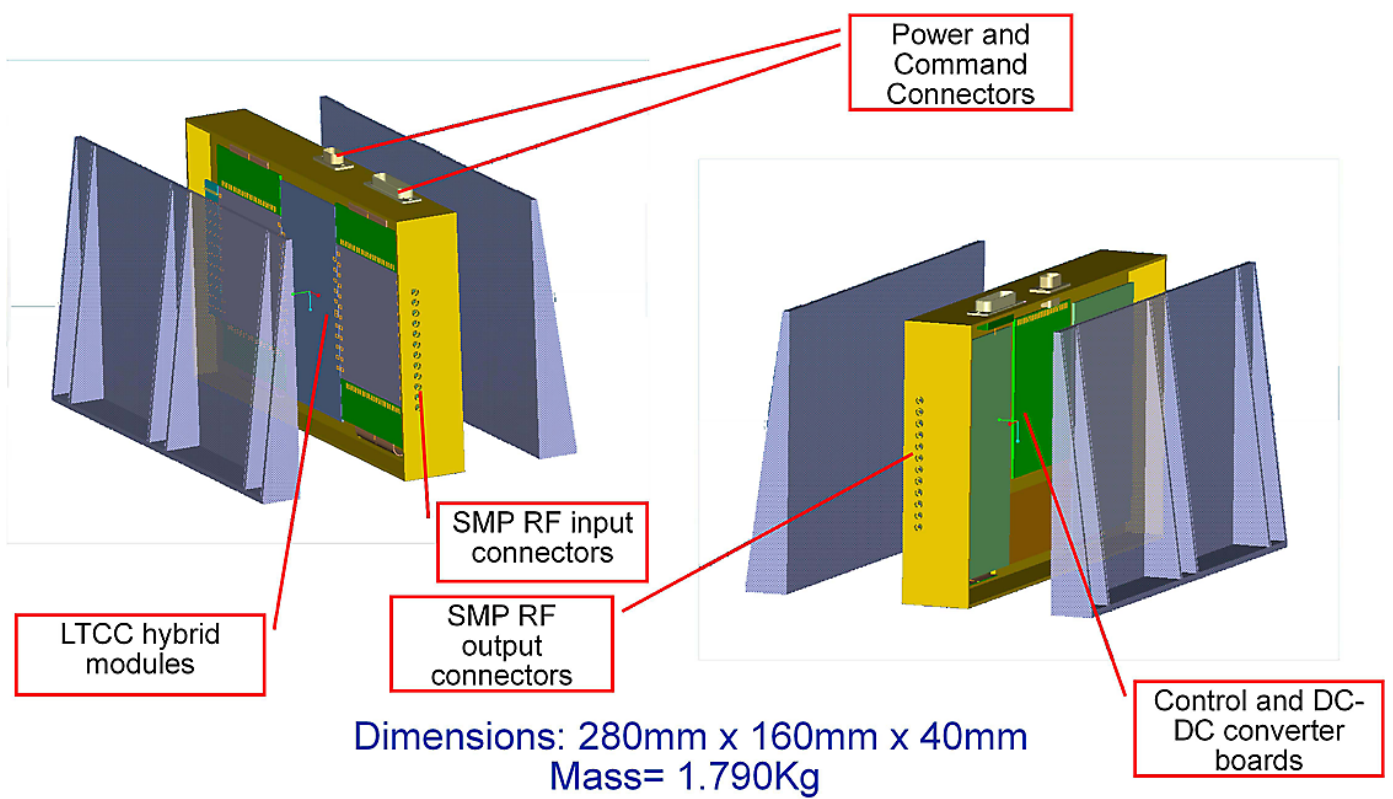

(a) Planar Benes network

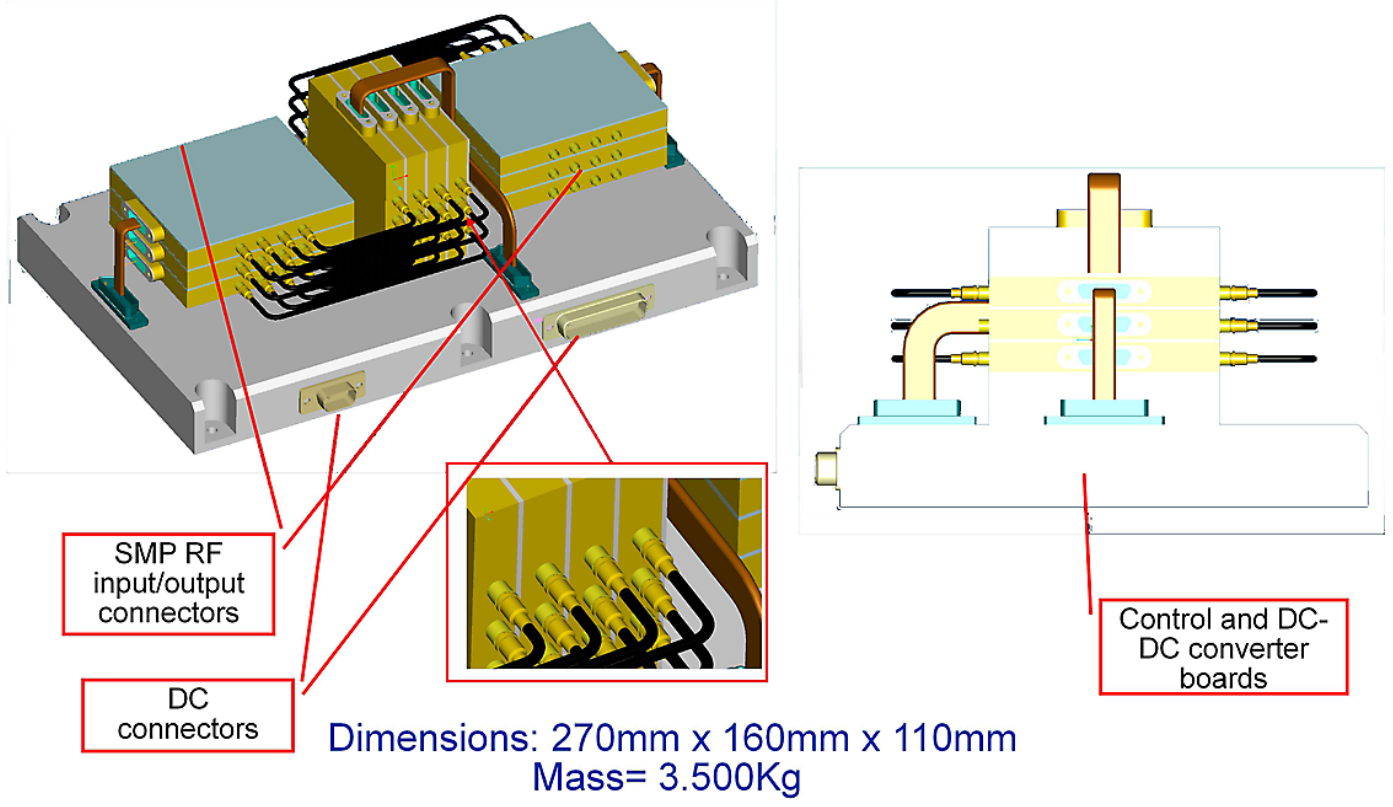

(b) 3D Clos Benes network

Fig. 2. Physical realization of planar Beneš network and 3-D Beneš network.

\subsubsection{RF connection}

The present $12 \times 12$ switch matrix adopts LTCC technology to construct multi-level circuits which enable the RF lines to be crossed without interfering each other. Figure 3 shows the LTCC board layouts which are designed for the $12 \times 12$ planar Beneš network. For the maximally feasible size of the LTCC board is $8.4 \mathrm{~cm}$ by $8.4 \mathrm{~cm}$, the entire network cannot be realized in the single board. Therefore, the full $12 \times 12$ network is splitted in 4 sections and each section is constructed on a different LTCC board of feasible size. Between LTCC boards, bond ribbons connect the RF circuits. Generally, bond ribbon connections are robust and immune against shocks and vibrations, which is critical for the space applications because the device should survive under the launching condition. Figure 4 shows how the DPDT switch is mounted 


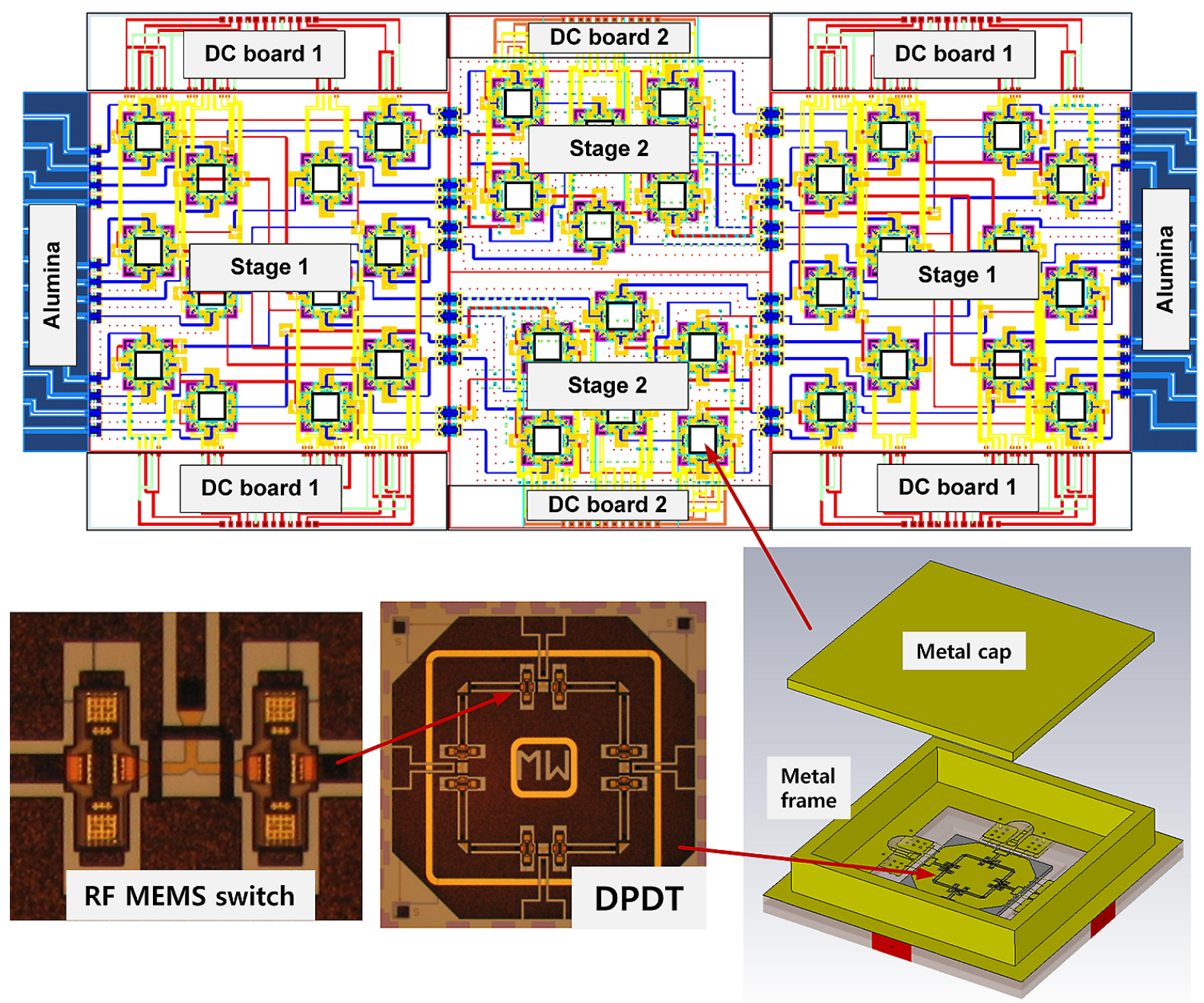

Fig. 3. $12 \times 12$ planar Beneš switch matrix layout and DPDT switch mount.

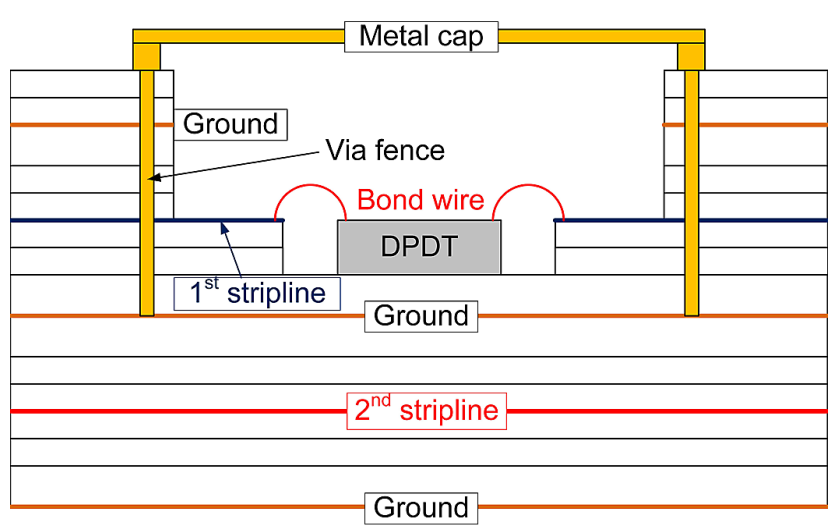

Fig. 4. DPDT switch mount on LTCC cavity.

on the LTCC board and electrically isolated from the environment. The DPDT switch is mounted in the deep LTCC cavity, where the switch circuit is located deep further to the level of the 1st stripline (SL) level on LTCC. The top and bottom layers of the switching element are grounded and connected with several vias. Consequently, each DPDT switch is capsulated by grounds and via fences, which suppress coupling between DPDTs. In the RF line design, every horizontal and vertical transition is optimized to have minimum return loss (RL) and insertion loss (IL). Additionally, metal vias are inserted between all SLs to suppress line couplings. On the input/output sections of the LTCC boards, the alumina boards are located. The reason of using alumina boards is to avoid physical collisions between LTCC boards and RF pin connectors on the housing during LTCC board installation.

\subsubsection{DC connection}

On the side (top and bottom side of the layout in Fig. 3) of the RF boards, 6 DC boards are connected. These DC boards are to decrease the number of DC connects. The single DPDT switch needs 4 DC contacts to control its $8 \mathrm{RF}$ 


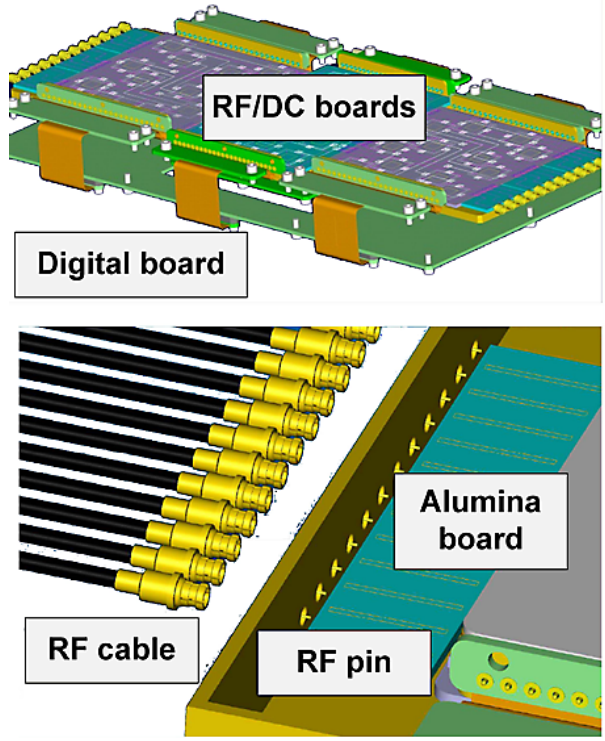

Fig. 5. Physical assembly of $12 \times 12$ network.

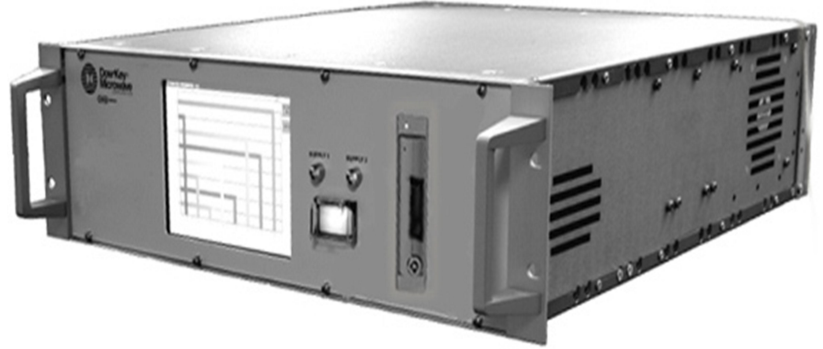

Fig. 6. Commercially available $12 \times 12$ Electromechanical RF Switching System (DowKey Microwave, 2012).

MEMS switches. And 36 DPDT switches are used for the entire network construction. This means, 144 DC contacts should be designed and simultaneously controlled. In this work, the number of DC contacts is decreased by binding DC contact pairs which are commonly activated during operation. This can also be done in the DPDT switch level. But the simpler approach is to bind common DC connects in the board level, which is done in the DC board. As a result, the number of DC contacts is reduced to 72 .

\subsection{Physical assembly}

Figure 5 shows the entire physical assembly of the $12 \times 12$ switching matrix. RF and DC boards are installed on the metal housing. Under the RF and DC boards, a digital board to generate switch matrix control signals is assembled and connected to the DC ports. The circuits on the RF boards are connected to the RF cables through RF pins. The connection between RF board circuits and RF pins are achieved by bond ribbons for high shock and vibration immunity.

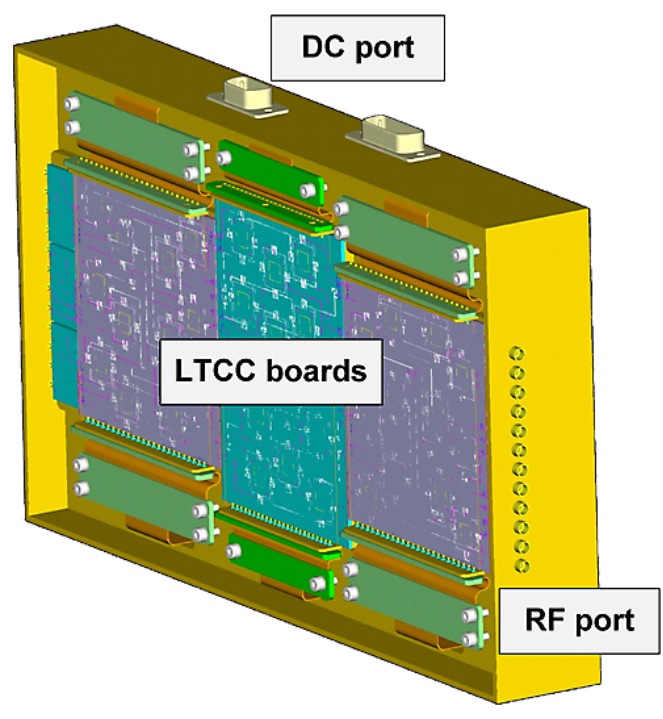

As described in Fig. 1, the present switch matrix assembly has a $28 \mathrm{~cm} \times 12 \mathrm{~cm} \times 2 \mathrm{~cm}$ size and a $1.79 \mathrm{~kg}$ weight. Figure 6 shows the commercially provided $12 \times 12$ mechanical switching system (DowKey Microwave, 2012). Its size is $48.3 \mathrm{~cm} \times 63.5 \mathrm{~cm} \times 17.8 \mathrm{~cm}$ and the weight is $38.56 \mathrm{~kg}$. Even after considering it has local control systems and a redundant power supply, it is much bigger and heavier than the proposed $12 \times 12$ RF MEMS switch matrix.

\subsection{Network control}

A high-order switch matrix needs a specific control algorithm. To control a $12 \times 12$ switch network, the 144 switch control inputs should be simultaneously assigned. Moreover, the number of feasible switch state configurations is 479001600 (=12!), which cannot be easily assigned by manual way or look-up tables. Therefore a switch matrix need the specific algorithm which can easily calculate the switch state and generate corresponding control inputs according to the given port assignments. Many network control algorithms have been reported, such as Looping algorithm, mMatching algorithm, Euler partition algorithm, Karol's algorithm or Frame-based matching algorithm, etc. In this work, a Looping algorithm is adopted to control the planar Bene $\breve{s}$ network. A Looping algorithm is a simple sequential optimal matching algorithm. It is simple and has a low complexity. The constraint of this algorithm is that the network should have two center modules and input/output modules have to be $2 \times 2$. In the present work, DPDT (Double-Pole Double-Throw) RF MEMS switches, which have $2 \times 2$ size, are used and the center stage consists of two identical modules, which fulfills the constraint of the Looping algorithm. (Chao and Liu, 2007). When the port assignments are given, 


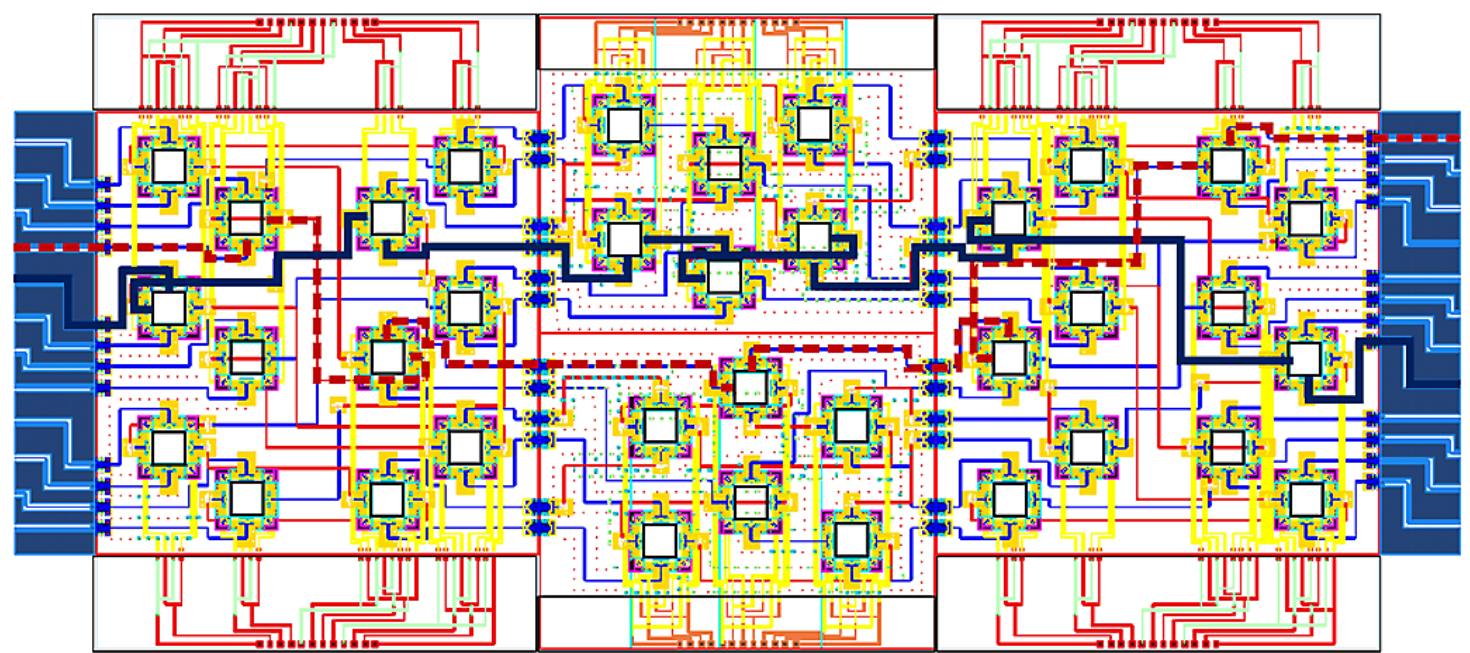

=- = $=$ Best case (5 DPDT switching, Least SL-SL transitions)

Worst case (7 DPDT switching, Most SL-SL transitions)

Fig. 7. Test line select.

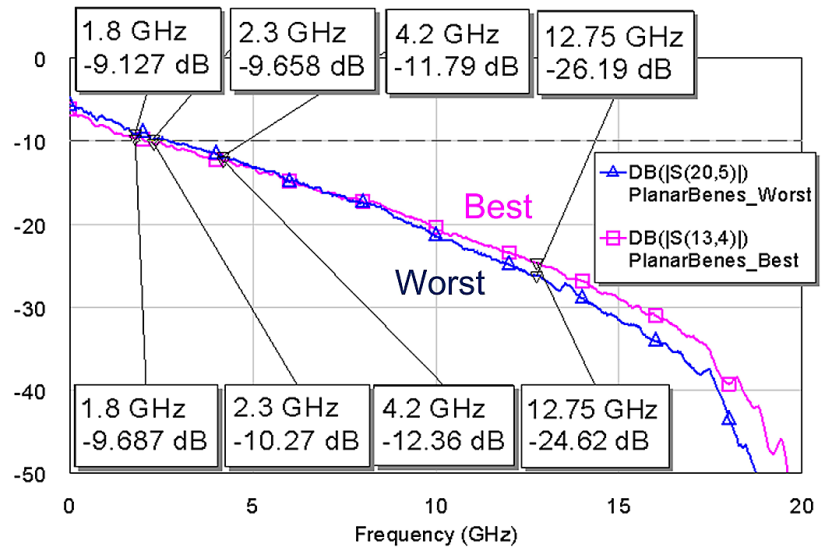

Fig. 8. Simulated IL of $12 \times 12$ network.

the corresponding switch states are calculated and switch control inputs are generated to be delivered to the digital board in the switch matrix (see Fig. 5). The digital board receives calculated DC inputs and controls high-voltage (typ. $60 \mathrm{~V})$ drivers which practically activate RF MEMS switches.

\subsection{RF signal transition performance}

Unlike the $16 \times 16$ network, the $12 \times 12$ network is not symmetric, which means the number of DPDT switches is different according to the path assignment. To simulate the RF signal transition performance, two paths are selected as the best and worst cases in terms of the DPDT switching, layerto-layer transition number and line lengths. Figure 7 shows the selected paths. Because the network is large and includes

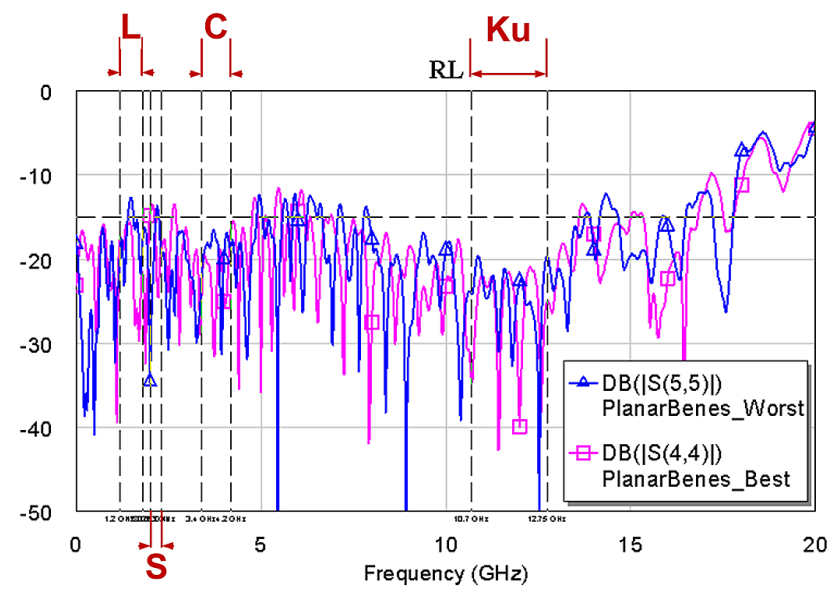

Fig. 9. Simulated RL of $12 \times 12$ network.

several complicated transitions, it is difficult to simulate the whole structure by full wave simulators. Therefore the detailed transition parts are full wave simulated and their results are combined with circuit simulators. The frequency band definitions are based on the commercial broadcasting satellite up/down link standards, which are $\mathrm{L}$ band (1.2-1.8 $\mathrm{GHz}), \mathrm{S}$ band (2.025-2.3 GHz), C band (3.4-4.2 GHz) and $\mathrm{Ku}$ band (10.7-12.75 GHz).

\subsubsection{Insertion loss (IL) and Return loss (RL)}

As the $12 \times 12$ network is not symmetric, the IL is different according to the path assignment. This is not preferred in satellite application because the switch matrix should be 


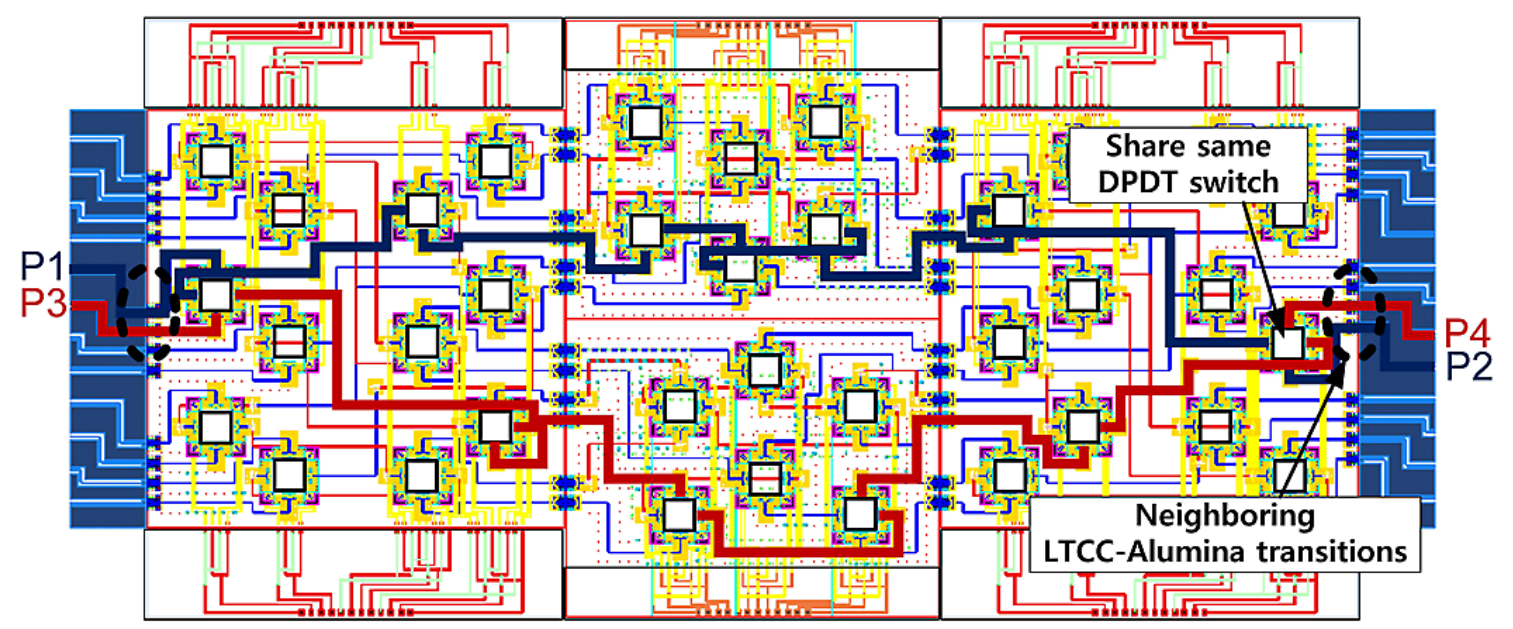

(a) Path coupled at the LTCC-Alumina transition

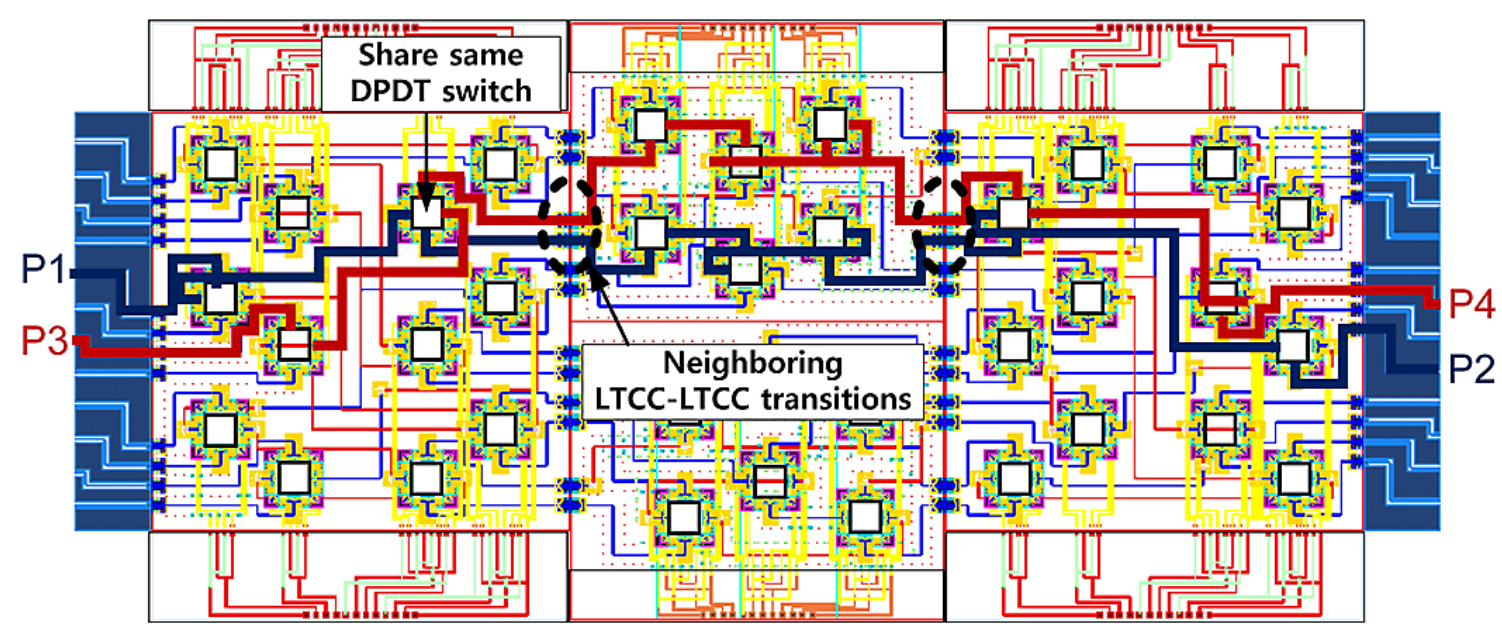

(b) Path coupled at the LTCC-LTCC transition

Fig. 10. Path coupling cases in the network.

transparent in terms of the RF signal transition. This means ILs should be the same for all connections regardless of the input/output port assignment. In the work, those differences are balanced by inserting resistive materials to the path which has less switching stages compared to the other paths. The resistivity and the size are carefully selected not to make severe RL increases. Figure 8 shows that the ILs are well-balanced and below $-12 \mathrm{~dB}$ up to $\mathrm{C}$ band. The main IL contributions are long transmission lines and the high number of switches, which cannot be easily decreased. However, the IL can be compensated by putting amplifiers in the output stage. Generally, high order switch matrices have high linearity amplifiers to compensate system IL as the commercially available switch matrix presented in Fig. 6. Concerning RL, the present matrix has some temporal peaks, but in general RL is below $-15 \mathrm{~dB}$ up to $\mathrm{Ku}$ band (see Fig. 9). This is better than the commercially available $12 \times 12$ mechanical switch ma- trix which has about $-7 \mathrm{~dB} \mathrm{RL}$ at $\mathrm{Ku}$ band (DowKey Microwave, 2012).

\subsubsection{Isolation}

Circuit simulators cannot effectively simulate the isolation of the network as they only consider the cascaded sub-circuit parameters without considering interferences between surrounding components. Therefore proper test structure definitions and approximations are to be involved to estimate the isolation. Generally, couplings mainly occur in the DPDT switch. In the planar Beneš network, the paths share an identical DPDT switch by two times at the maximum, which is the worst case for the isolation. As the worst case, the two paths in Fig. 10 are selected, which are coupled two times at the DPDT switch. Line couplings are accounted by inserting coupled lines at every switching stage. The coupled lines have the feasible maximum length in the network and 


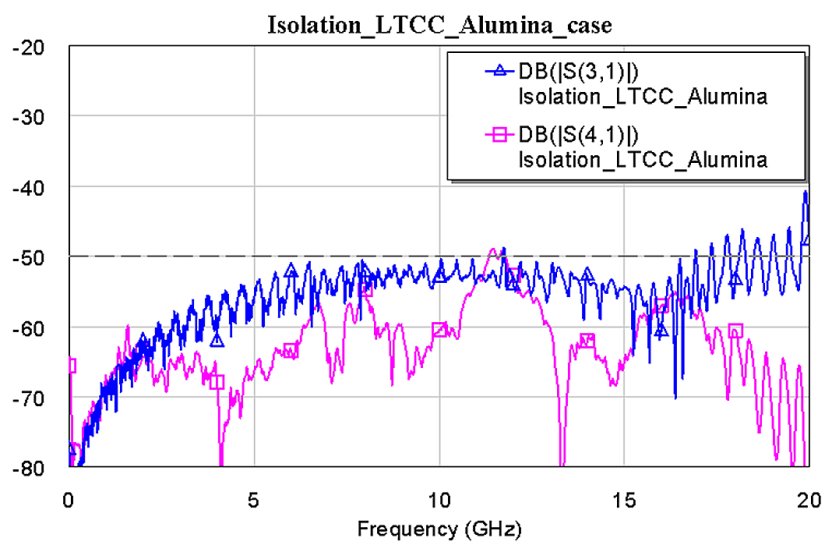

(a) Coupled in LTCC-Alumina transition

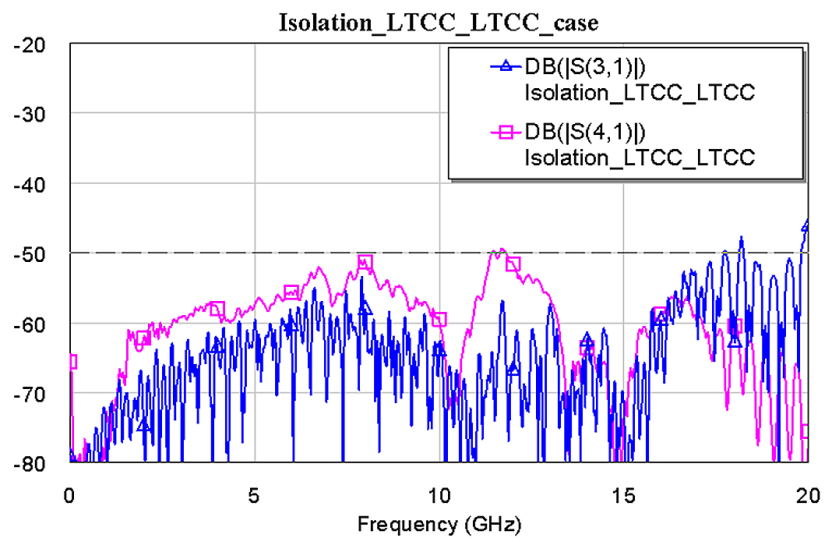

(b) Coupled in LTCC-LTCC transition

Fig. 11. Simulated isolation of $12 \times 12$ network.

via fences are inserted between lines. A coupling also occurs at the board-to-board transitions, LTCC-LTCC and LTCCAlumina. In terms of the worst isolation, the couplings in the board-to-board transitions can be separated into two cases. One case is when the LTCC-Alumina transition ports are two times neighbored (see Fig. 10a). Another case is that LTCCLTCC transition paths are neighbored and two times coupled (see Fig. 10b). Due to the nature of the Beneš network, those two cases can not happen at the same time. Figure 11 shows the isolation of the $12 \times 12$ network. For all frequency bands of interest, the isolation is better than $-50 \mathrm{~dB}$. The commercially available $12 \times 12$ mechanical switch matrix provided in DowKey Microwave (2012) has a $-50 \mathrm{~dB}$ input/output isolation, which is comparable to the present matrix isolation.

\subsection{3 $4 \times 4$ switch matrix fabrication and measurement}

The $4 \times 4$ switch matrix in Fig. 12 is to test and verify the design process of the $12 \times 12$ switch matrix. The $4 \times 4$ matrix is the simplified form of the $12 \times 12$ matrix and fabricated by the same design process and technologies. There are some differences from the $12 \times 12$ matrix, which are mainly

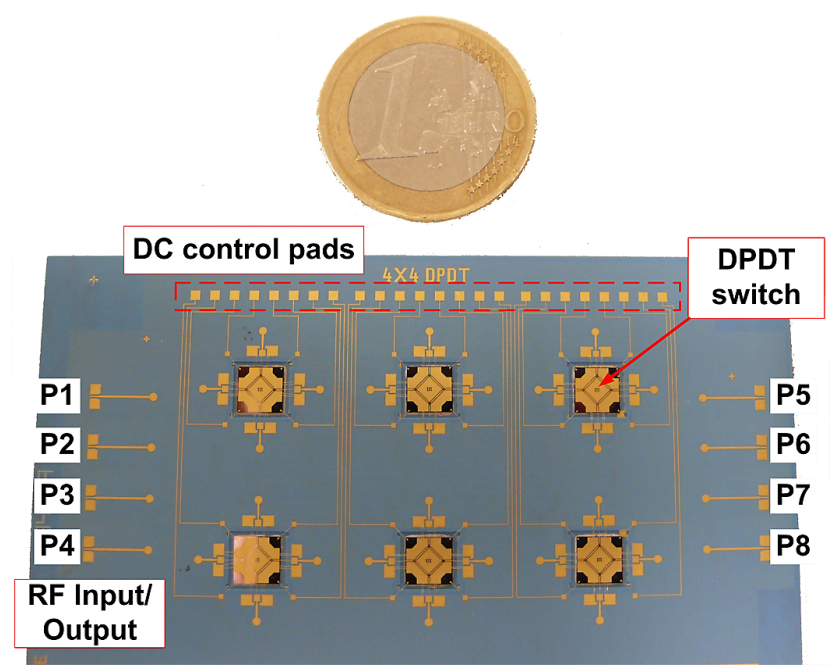

Fig. 12. $4 \times 4$ switch matrix using RF MEMS switches.

from the design developments after the $4 \times 4$ fabrication has started. The main difference is the mounting level of DPDT switches. In the $4 \times 4$ matrix, the DPDT switch is mounted on the higher level, where the DPDT circuit is the level of microstip line (MSL) of the LTCC board. Therefore the matrix has not only SL-SL transitions but also microstrip line (MSL) to SL transitions which are not required in the $12 \times 12$ matrix. Additionally, in the $4 \times 4$ matrix, no bondwire compensation structures which suppress the high bondwire inductance and high RL are made on the DPDT switch. Moreover, the DPDT switch and LTCC circuits are connected by a single bondwire. In the $12 \times 12$ matrix, a double bondwire connection is adopted and bondwire compensation structures are designed on the DPDT switch side as well. Another difference is that the transmission lines of the $4 \times 4$ matrix are closely distanced compared to the $12 \times 12$ matrix and no via fences are inserted. However, the performance and comparison to the simulations is still of interest because the same design methods and technologies are used. Figure 13 shows the measured performance of the $4 \times 4$ matrix and the comparisons to the simulations. The left three graphs show the IL, RL and Isolation when the port 1 and 5 are connected, and the right three are from the connection of port 4 and 5. To measure the isolation, the neighboring port 6 is measured. The measurements agree well to the simulations even though the transition performance is not as good as $12 \times 12$ matrix's due to the design differences as already noted. Isolation deviations at the high frequency are mainly due to the nature of the circuit simulator which does not account for the component coupling. 

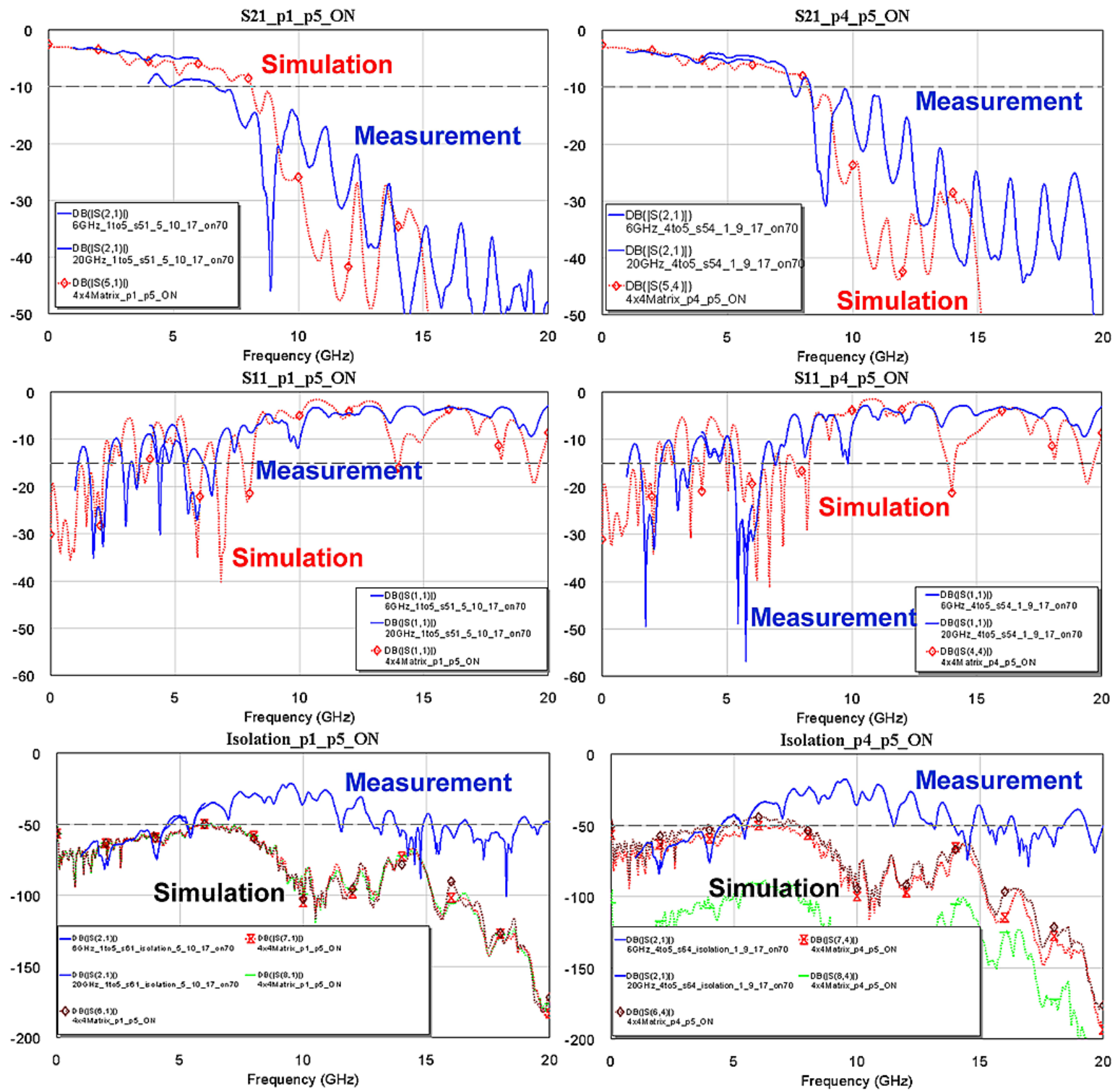

Fig. 13. Measured performance of $4 \times 4$ matrix and comparison to the simulations.

\section{Conclusions}

In this work, a $12 \times 12$ switch matrix using RF MEMS and LTCC technologies is designed based on the planar Beneš network controlled by Looping algorithms. For the switching components, RF MEMS DPDT switches are used and they are connected by multi-layer transmission lines fabricated by LTCC technology. The matrix shows $-12 \mathrm{~dB}$ IL up to $\mathrm{C}$ band and below $-15 \mathrm{~dB}$ RL up to Ku band. Isolation better than $-50 \mathrm{~dB}$ is also expected at the frequency bands of interest. Compared to the $12 \times 12$ mechanical switch ma- trix which is commercially available, the designed $12 \times 12$ switch matrix has less than $10 \%$ of size and volume and better RL with comparable isolation. For the verification of the matrix performance, a $4 \times 4$ switch matrix with the same design process and technology is fabricated and measured. The measurements and the simulations agree well each other, which makes the $12 \times 12$ switch matrix design process and technology more confident. 
Acknowledgements. The work has been carried out in the frame of the ESA Project No AO/1-5136/06/NL/GLC. The authors will thank Francois Deborgies from ESA/ESTEC for his support and suggestions.

\section{References}

Chang, C. and Melhem, R.: Arbitrary Size Benes Networks, J. Parallel Process. Lett., 7, 279-284, 1997.

Chao, H. J. and Liu, B.: High performance switches and routers, John Wiley \& Sons, Inc., Hoboken, New Jersey, USA, 2007.
De Angelis, G., Lucibello, A., Proietti, E., Marcelli, R., Bartolucci, G., Casini, F., Farinelli, P., Mannocchi, G., Di Nardo, S., Pochesci, D., Margesin, B., Giacomozzi, F., Vendier, O., Kim, T., and Vietzorreck, L.: RF MEMS ohmic switches for matrix configurations, Int. J. Microw. Wirel. Technol., 4, 421-433, doi:10.1017/S1759078712000074, 2012.

Electromechanical - RF Switching System: http://www.dowkey. com, last access: 7 December 2012.

Imanaka, Y.: Multilayered Low Temperature Cofired Ceramics (LTCC) Technology, Springer Science + Business Media, Inc., Boston, USA, 2005.

Yeh, Y.-M. and Feng, T.: On a class of rearrangeable networks, IEEE T. Comput., 41, 1361-1379, doi:10.1109/12.177307, 1992. 